\title{
DISTRIBUIÇÃO ESPACIAL DE FERRO, COBRE E CHUMBO EM SEDIMENTOS DE MANGUEZAL EM UM GRADIENTE DE DEGRADAÇÃO NA BAÍA DE GUANABARA (ESTADO DO RIO DE JANEIRO)
}

\author{
Anderson C. Borges*, Jailson C. Dias e Wilson Machado e Sambasiva R. Patchineelam \\ Departamento de Geoquímica, Instituto de Química, Universidade Federal Fluminense, Outeiro de São João Batista, s/n, \\ 24020-007 Niterói - RJ, Brasil \\ Silvia M. Sella \\ Departamento de Química Analítica, Instituto de Química, Universidade Federal Fluminense, 24020-007 Niterói - RJ, Brasil \\ Recebido em 23/11/05; aceito em 7/4/06; publicado na web em 11/8/06
}

\begin{abstract}
SPATIAL DISTRIBUTION OF IRON, COPPER AND LEAD IN MANGROVE SEDIMENTS IN A DEGRADATION GRADIENT IN GUANABARA BAY (RIO DE JANEIRO STATE). Iron, copper and lead distribution was evaluated in sediment cores from a disturbed mangrove area in Guanabara Bay: a core from a seaward site where mangrove vegetation was removed 20 yr before sampling (MD); a core from an intermediate site with dead vegetation, apparently due to insect attack (MP), and a core from a landward site with living vegetation (MV). Metal concentrations showed increasing values seaward while organic matter content showed an inverse trend, displaying a negative correlation with metals. This unusual correlation indicates opposite sources, since metals come from the bay and the main OM origin is probably degraded mangrove vegetation. Plant cover loss seems to be a critical factor affecting metal accumulation, particularly due to changes in OM input.
\end{abstract}

Keywords: trace metals; contamination; mangrove sediments.

\section{INTRODUÇÃO}

Depois da revolução industrial houve um grande incremento no lançamento de metais-traço em ecossistemas aquáticos, sem tratamento prévio adequado ${ }^{1}$. Entretanto, ecossistemas como os manguezais podem constituir verdadeiras barreiras biogeoquímicas na interface continente-oceano, retendo contaminantes metálicos em seus sedimentos, como vem sendo indicado por estudos em diversas áreas tropicais e subtropicais ${ }^{2-4}$. No entanto, é importante considerar que tais metais têm diferentes comportamentos geoquímicos, que podem favorecer sua retenção ou sua liberação pela fase sólida dos sedimentos para as águas costeiras. Além disso, a variabilidade espacial e temporal no seu aporte e na composição dos sedimentos pode influenciar a distribuição dos metais-traço ${ }^{5-7}$.

Considerando que sedimentos de manguezal favorecem o desenvolvimento de condições predominantemente redutoras e a acumulação de metais-traço na forma de sulfetos metálicos e de complexos com a matéria orgânica ${ }^{2,8,9}$, como vem sendo indicado para diferentes manguezais na Baía de Guanabara (Estado do Rio de Janeiro $)^{6,10,11}$, pode-se esperar que estas duas fases sedimentares sejam fatores importantes para determinar a "reatividade" dos metais, isto é, o nível de acumulação em formas mais susceptíveis a transformações químicas e potencialmente mais biodisponíveis. Por ex., metais-traço são reconhecidos por sua tendência em apresentar diferentes níveis de incorporação pela pirita (o sulfeto metálico cristalino mais estável) e por sulfetos amorfos ou pouco cristalizados (considerados reativos), como mostrado pelos dados compilados por Morse e Luther ${ }^{12}$. Além disso, Andrade e Patchineelam ${ }^{13}$ demonstraram que metais como $\mathrm{Cu}, \mathrm{Ni}$ e $\mathrm{Cr}$ podem apresentar maior acumulação em fases reativas de sedimentos de manguezal com condições mais oxidantes que em sedimentos de manguezal com condições mais redutoras.

A Baía de Guanabara possui $384 \mathrm{~km}^{2}$ de área, sendo um corpo

*e-mail: borgesadc@yahoo.com.br d'água parcialmente eutrofizado, que recebe esgotos de aproximadamente 7,8 milhões de habitantes e é rodeado por mais de 6000 indústrias ${ }^{14}$. Nas últimas cinco décadas este ecossistema tem sido alvo de diversos impactos antrópicos, tais como aporte de efluentes domésticos e rejeitos industriais, desmatamento da vegetação de manguezal para extração de madeira, aterros, ocupação desordenada de terras públicas e derramamentos de óleo, os quais implicam na perda da qualidade ambiental e na mortandade da biota local. Contudo, existem áreas remanescentes de florestas de manguezal que podem estar retendo contaminantes metálicos nos sedimentos, contribuindo para moderar o seu possível efeito negativo nas águas da baía.

A área fisiográfica em estudo está situada no noroeste da Baía de Guanabara, no município de Mauá (Figura 1). Embora existam relativamente poucas áreas de manguezal da Baía de Guanabara que tenham sido estudadas quanto à acumulação de metais-traço nos sedimentos $^{6,10,11}$, existem estudos mais extensos ao longo da área coberta pelas águas da baía sobre a acumulação de metais em sedimentos ${ }^{15,16}$, possibilitando a avaliação de como cada área se encontra dentro do contexto geral da contaminação desta baía. Rebello et al..$^{15}$ destacaram que a região do rio Estrela, onde se encontra a área de estudo, sofre uma elevada contaminação por $\mathrm{Cu}$ de origem industrial.

Neste trabalho buscou-se investigar a distribuição dos metais $\mathrm{Fe}, \mathrm{Cu}$ e $\mathrm{Pb}$ em sedimentos ao longo de uma área de manguezal com diferentes níveis de degradação, localizada no noroeste da Baía de Guanabara.

\section{PARTE EXPERIMENTAL}

Em fevereiro de 2004 foram coletados três testemunhos na área entre-marés de Mauá: um testemunho proveniente da planície de lama onde a floresta de manguezal sofreu desmatamento, segundo comunicações pessoais de moradores do local, cerca de 20 anos antes da coleta (testemunho MD); um testemunho onde havia uma floresta morta cerca de 2 anos antes da coleta, aparentemente devido ao ata- 
que de pragas (testemunho MP) e, um testemunho onde havia um remanescente de floresta de Laguncularia racemosa (testemunho MV). Como mostrado na Figura 1, os pontos de coleta apresentam uma distribuição aproximadamente perpendicular à linha de costa, sendo a posição do testemunho MV a mais próxima ao continente e a posição do testemunho MD a mais próxima à baía. Os testemunhos foram seccionados em intervalos de $2 \mathrm{~cm}$ até $10 \mathrm{~cm}$ de profundidade e os sedimentos foram secos em estufa $\left(50{ }^{\circ} \mathrm{C}\right.$ por $\left.72 \mathrm{~h}\right)$ e macerados em gral de ágata, a fim de serem homogeneizados. A extração dos metais foi realizada com $0,400 \mathrm{~g}$ de sedimento seco, sob contínua agitação em uma solução de $\mathrm{HCl} 1 \mathrm{~mol} \mathrm{~L}^{-1}$ durante $1 \mathrm{~h}$, com o objetivo de extrair a fração reativa dos metais, que representa os metais presentes em óxidos e sulfetos amorfos ou pouco cristalizados, carbonatos e matéria orgânica lábil, assim como os metais adsorvidos às frações orgânicas e minerais dos sedimentos. As concentrações de $\mathrm{Fe}, \mathrm{Cu}$ e $\mathrm{Pb}$ foram determinadas por espectrofotometria de absorção atômica ${ }^{17}$, empregando-se um espectrofotômetro Varian, modelo SpectraA-300. Duplicatas de $40 \%$ do total das amostras foram utilizadas para avaliar a precisão das análises. Foram observadas precisões de $4 \%$ para $\mathrm{Fe}, 11 \%$ para $\mathrm{Cu}$ e $8 \%$ para $\mathrm{Pb}$.

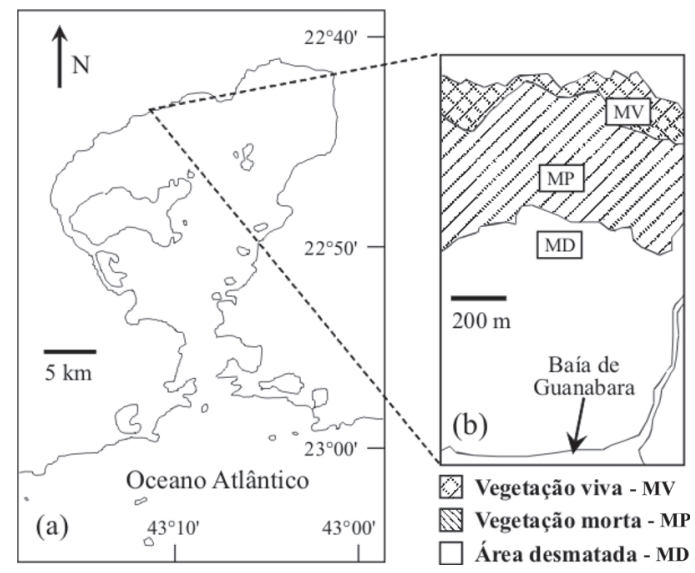

Figura 1. Mapa de localização do manguezal de Mauá (a) e dos pontos de amostragem na área em estudo (b) na Baía de Guanabara, Rio de Janeiro

Para caracterização dos ambientes sedimentares e de possíveis fatores de influência sobre a distribuição dos metais, a quantificação do conteúdo de matéria orgânica (MO) foi realizada de acordo com o método da perda por ignição $\left(450{ }^{\circ} \mathrm{C}\right.$ por $5 \mathrm{~h}$ ), enquanto o conteúdo de sedimentos finos (argilas e siltes) foi determinado após peneiramento em malha de $63 \mu \mathrm{m}$, com posterior secagem das amos$\operatorname{tras}\left(50{ }^{\circ} \mathrm{C}\right.$ por $\left.72 \mathrm{~h}\right)$.

\section{RESULTADOS E DISCUSSÃO}

Os valores médios, máximos e mínimos dos resultados obtidos para concentrações dos metais, conteúdo de matéria orgânica (MO) e conteúdo de sedimentos finos $(<63 \mu \mathrm{m})$ nos três testemunhos analisados encontram-se discriminados na Tabela 1 , enquanto a variabilidade vertical destes resultados está apresentada nas Figuras 2 e 3 .

Os sedimentos apresentaram uma textura predominantemente fina (com mais de $60 \%$ de argilas e siltes) e um elevado conteúdo de MO (19 a 42\%) em todos os pontos de amostragem (Tabela 1), além de apresentarem coloração cinza, típica de sedimentos redutores com presença de sulfetos de ferro ${ }^{18}$, como é esperado para áreas entremarés desta região degradada da Baía de Guanabara ${ }^{6}$.

Pode-se perceber uma forte tendência de aumento na concentração de todos os metais entre os testemunhos estudados na se-
Tabela 1. Médias das concentrações dos metais e dos conteúdos de matéria orgânica (MO) e de sedimentos finos $(<63 \mu \mathrm{m})$ nos testemunhos estudados (valores mínimos e máximos estão apresentados entre parênteses)

\begin{tabular}{lcccc}
\hline & Unidade & $\begin{array}{c}\text { Testemunho } \\
\text { MD }\end{array}$ & $\begin{array}{c}\text { Testemunho } \\
\text { MP }\end{array}$ & $\begin{array}{c}\text { Testemunho } \\
\text { MV }\end{array}$ \\
\hline $\mathrm{Fe}$ & $\mathrm{mg} \mathrm{g}^{-1}$ & 21,3 & 6,8 & 3,9 \\
& & $(18,3-23,4)$ & $(5,88-8,83)$ & $(2,38-5,35)$ \\
$\mathrm{Cu}$ & $\mu \mathrm{g} \mathrm{g}^{-1}$ & 65,3 & 9,5 & 1,6 \\
& & $(52,1-75,0)$ & $(5,27-12,5)$ & $(0,36-4,46)$ \\
$\mathrm{Pb}$ & $\mu \mathrm{g} \mathrm{g}^{-1}$ & 35,3 & 24,1 & 11,5 \\
& & $(32,7-39,2)$ & $(22,9-25,4)$ & $(9,77-12,7)$ \\
$\mathrm{MO}$ & $\%$ & 20,8 & 36,5 & 40,5 \\
& & $(19,2-25,3)$ & $(31,1-38,7)$ & $(37,5-42,5)$ \\
$<63 \mu \mathrm{m}$ & $\%$ & 80,4 & 78,6 & 66,8 \\
& & $(69,7-90,3)$ & $(74,0-85,3)$ & $(63,9-74,3)$ \\
\hline
\end{tabular}
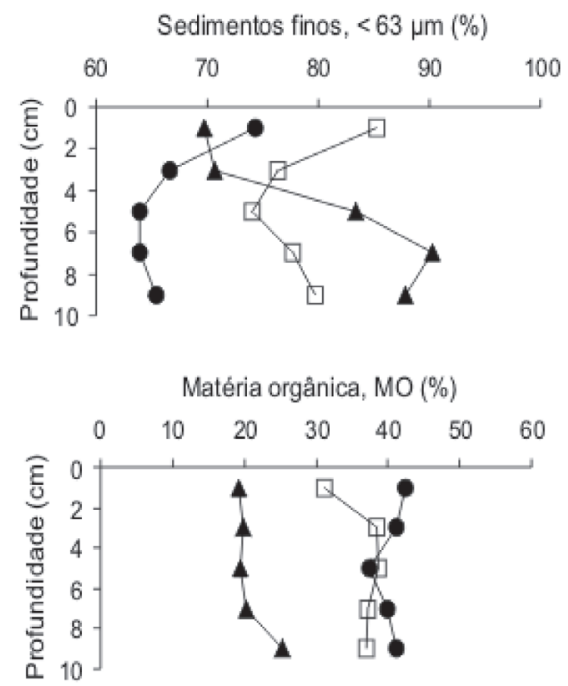

Figura 2. Distribuição vertical dos conteúdos de sedimentos finos $(<63 \mu \mathrm{m})$ e de matéria orgânica (MO) nos testemunhos MD (triângulos), MP (quadrados) e MV (círculos), coletados em fevereiro de 2004, no manguezal de Mauá, Baía de Guanabara

quiência MD > MP > MV (Tabela 1), com os maiores valores obtidos no ponto de amostragem correspondente à área mais degradada e próxima à baia. Deve-se destacar a concentração do $\mathrm{Cu}$, que apresentou valores até duas ordens de grandeza superiores no testemunho MD quando comparado ao testemunho MV. Este gradiente de concentração significativo pode ter sido determinado por diferentes aportes de materiais pelas águas de maré e processos biogeoquímicos, como discutido abaixo.

Apesar do $\mathrm{Pb}$ não apresentar nenhuma tendência definida nos valores de concentração com a profundidade, os resultados obtidos para concentrações de $\mathrm{Cu}$ mostraram redução com a profundidade. Esta mesma tendência foi verificada para os teores de Fe nos testemunhos MD e MV (Figura 3).

Enquanto a concentração de todos os metais estudados apresentou considerável aumento no local mais próximo à baía, o teor de MO acumulada apresentou uma tendência oposta, com os maiores valores obtidos no ponto mais próximo ao continente. O conteúdo de sedimentos finos não apresentou uma tendência tão forte quanto a dos metais, mas também apresentou um aumento no sentido da baía (Tabela 1).

Nos resultados obtidos neste estudo (Figura 2) não foi observada uma variação vertical substancial no conteúdo de MO, exceto por menor acumulação na camada superior do testemunho MP e 

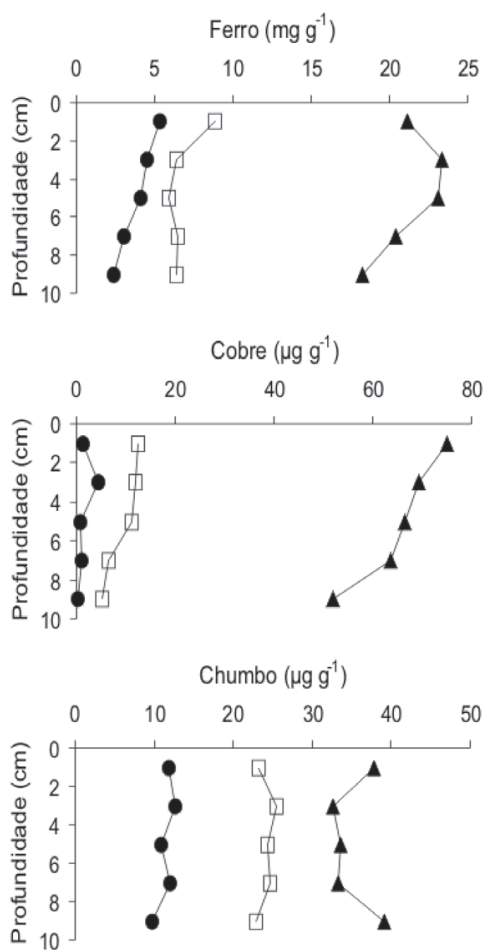

Figura 3. Distribuição vertical das concentrações reativas de $\mathrm{Fe}, \mathrm{Cu}$ e $\mathrm{Pb}$ nos testemunhos MD (triângulos), MP(quadrados) e MV (círculos), coletados em fevereiro de 2004, no manguezal de Mauá, Baía de Guanabara

maior acumulação na camada inferior do testemunho MD. O conteúdo de sedimentos finos apresentou tendências distintas entre os testemunhos MD (com menor acumulação na superfície) e os testemunhos MP e MV (com menor acumulação logo abaixo da superfície e valores máximos no topo).

Diversos estudos anteriores têm indicado correlações positivas significativas entre as concentrações de metais-traço e os conteúdos de MO e sedimentos finos em áreas de manguezal ${ }^{2,5,8}$. Considerando-se os resultados obtidos para todos os testemunhos, apenas $\mathrm{Pb}$ apresentou uma correlação positiva significativa com o conteúdo de sedimentos finos $(\mathrm{r}=0,65 ; \mathrm{p}<0,01)$. Porém, ocorreram correlações negativas significativas do conteúdo de MO com as concentrações de $\mathrm{Fe}(\mathrm{r}=-0,98 ; \mathrm{p}<0,001), \mathrm{Cu}(\mathrm{r}=-0,97 ; \mathrm{p}<0,001)$ e $\mathrm{Pb}(\mathrm{r}=-0,87 ; \mathrm{p}<0,001)$, em oposição aos resultados geralmente encontrados na literatura. Estas correlações inversas podem tornarse ainda menos esperadas quando se considera que a geoquímica do $\mathrm{Cu}$ na Baía de Guanabara é reconhecidamente associada à formação de complexos orgânicos na coluna d'água ${ }^{19}$, além da evidenciada associação de metais, como o Pb, e sulfetos em sedimentos desta baía ${ }^{20}$ ser dependente da geoquímica da matéria orgânica, que envolve processos como o sustento da sulfato-redução e da própria preservação de condições redutoras nos sedimentos.

Ao contrário do que geralmente ocorre em ambientes menos impactados, os resultados obtidos demonstram que a degradação da floresta de manguezal tem reflexos significativos na distribuição dos metais em sedimentos. Esta degradação provavelmente causou um incremento temporário no aporte de MO do manguezal para os sedimentos (pela decomposição de caules e/ou raízes, dependendo do local ter sido desmatado ou não), isto é, uma MO lenhosa e provavelmente pobre em metais, pois esta vegetação apresenta mecanismos adaptativos que moderam e até excluem a incorporação de metais potencialmente tóxicos ${ }^{10,21,22}$.

Desta forma, o perfil de distribuição de MO e metais observado neste estudo, em contraste com dados relatados na literatura, pode ser explicado pela ocorrência de diferentes fontes para estes constituintes sedimentares. Enquanto o aporte pelas águas de maré é a principal via de entrada de metais para a área de estudo (enriquecendo os sedimentos em metais reativos no sentido da baía), uma mudança na natureza da MO acumulada nos sedimentos, devida ao processo de degradação da vegetação, vem provavelmente contribuindo para a menor acumulação de metais reativos em direção ao continente.

Considerando que uma alteração qualitativa na $\mathrm{MO}$ seja um processo que ocorre na área de estudo, ela provavelmente está mascarando possíveis relações positivas entre a MO originalmente acumulada e os metais. Além disso, uma associação da perda da proteção física e da influência da cobertura vegetal sobre a acumulação e estabilização dos sedimentos ${ }^{23,24}$ com um aporte diferenciado de MO, provavelmente pode promover alterações na diagênese recente $\mathrm{e}$ nas relações entre os constituintes sedimentares estudados. Estes processos devem ocorrer de forma diferenciada entre os locais de coleta, mas são necessários estudos incluindo uma caracterização qualitativa da MO para se obter uma interpretação conclusiva.

Além da variabilidade no aporte, a variabilidade vertical e lateral no fracionamento entre fases reativas e refratárias dos metais nos sedimentos pode estar também contribuindo para determinar as tendências observadas ${ }^{13,18}$. Por ex., pode-se esperar que ocorra uma redução na fração reativa com o aumento na profundidade dos sedimentos. Isto devido a processos diagenéticos, que transformam os compostos metálicos reativos em estruturas mais refratárias ou fazem com que metais superficialmente adsorvidos sejam incorporados na estrutura de compostos refratários, como a própria pirita.

Este conjunto de processos resultou em um gradiente significativo das concentrações reativas dos metais estudados, que pode refletir também a contaminação da área. Perin et al. ${ }^{16}$ relataram que as medianas das concentrações de $\mathrm{Cu}$ e $\mathrm{Pb}$ (extraídas em solução concentrada de $\mathrm{HNO}_{3}$ ) para a área da Baía de Guanabara correspondem a 52,0 e 72,8 $\mu \mathrm{g} \mathrm{g}^{-1}$, respectivamente. As concentrações reativas de $\mathrm{Pb}$ na área de estudo (Tabela 1 ) foram de 2 a 7 vezes menores que a mediana indicada para a baía, mas as concentrações de $\mathrm{Cu}$ observadas variaram desde duas ordens de grandeza a menos a até valores ligeiramente acima (Tabela 1) da mediana indicada para a baía. Considerando que estas medianas incluem as concentrações de formas refratárias dos metais (extraídas em $\mathrm{HNO}_{3}$ concentrado), que não foram incluídas nas concentrações determinadas no presente estudo (extraídas em $\mathrm{HCl} 1 \mathrm{~mol} \mathrm{~L}^{-1}$ ), pode-se concluir que os sedimentos estudados atingiram níveis de contaminação de $\mathrm{Cu}$ seguramente acima daquele geralmente encontrado na área da Baía de Guanabara. Em comparação com outras áreas de manguezal na baía, as concentrações de $\mathrm{Cu}$ atingiram valores próximos ao extremo superior da variação de concentrações extraídas com soluções ácidas concentradas $\left(18,0 \text { a } 91,7 \mu \mathrm{g} \mathrm{g}^{-1}\right)^{10,11}$ e valores superiores a concentrações extraídas em água régia diluída (32 a 58 $\left.\mu \mathrm{g} \mathrm{g}^{-1}\right)^{6}$. As concentrações de $\mathrm{Pb}$ atingiram valores dentro da faixa de variação das concentrações obtidas em outras áreas de manguezal da baía com extrações ácidas concentradas $\left(20,0 \text { a 169,5 } \mu \mathrm{g} \mathrm{g}^{-1}\right)^{10,11}$.

Os resultados apontam sérios problemas de contaminação por $\mathrm{Cu}$ nos sedimentos da área desmatada nas últimas quatro décadas, de acordo com a taxa de sedimentação estimada em $0,24 \mathrm{~cm}^{2} \mathrm{ano}^{-1}$ para esta área ${ }^{25}$, calculada através do método de datação por ${ }^{210} \mathrm{~Pb}$.

\section{CONCLUSÃO}

Os resultados indicam que a poluição das águas da baía de Guanabara vem resultando em um elevado aporte de $\mathrm{Cu}$ e $\mathrm{Pb}$ para a área em estudo, onde a degradação da vegetação traz como conse- 
quiência uma alteração na acumulação de MO, que pode afetar significativamente a acumulação de metais.

Em contraste com outras áreas, foi observada uma relação de distribuição espacial inversa entre o conteúdo de $\mathrm{MO}$ e as concentrações de $\mathrm{Fe}, \mathrm{Cu}$ e $\mathrm{Pb}$, o que pode ser explicado pela ocorrência de diferentes fontes para estes constituintes sedimentares. A mudança na natureza da MO devida ao processo de degradação da vegetação vem, provavelmente, contribuindo para a menor acumulação de metais reativos no sentido do continente, o que pode estar alterando significativamente a geoquímica de metais-traço nos sedimentos estudados.

\section{AGRADECIMENTOS}

A. Borges e J. C. Dias agradecem o suporte financeiro da CAPES. W. Machado e S. Patchineelam agradecem o suporte financeiro da FAPERJ.

\section{REFERÊNCIAS}

1. Nriagu, J. O.; Pacyna, J. M.; Nature 1988, 33, 134.

2. Harbison, P.; Mar. Pollut. Bull. 1986, 17, 273.

3. Lacerda, L. D.; Rezende, C. E.; Aragon, G. T.; Ovalle, A. R. C.; Water, Air, Soil Pollut. 1991, 57/58, 513.

4. Alongi, D. M.; Wattayakorn, G.; Boyle, S.; Tirendi, F.; Payn, C.; Dixon, P.; Biogeochemistry 2004, 69, 105.

5. Tam, N. F. Y.; Wong, Y. S.; Mar. Pollut. Bull. 1995, 31, 254.
6. Machado, W.; Moscatelli, M.; Rezende, L. G.; Lacerda, L. D.; Environ. Pollut. 2002, 120, 455.

7. Silva, L. F. F.; Machado, W.; Lisboa Filho, S. D.; Lacerda, L. D.; Water, Air, Soil Pollut. 2003, 145, 67.

8. Soto-Jiménez, M. F.; Páez-Osuna, F.; Est. Coastal Shelf Sci. 2001, 53, 259.

9. Aragon, G. T.; Miguens, F. C.; Geo-Mar. Lett. 2001, 21, 157.

10. Machado, W.; Silva Filho, E. V.; Oliveira, R. R.; Lacerda, L. D.; Mar. Pollut. Bull. 2002, 44, 1277 .

11. Kehrig, H. A.; Pinto, F. N.; Moreira, I.; Malm, O.; Org. Geochem. 2003, 34,661 .

12. Morse, J. W.; Luther, G. W.; Geochim. Cosmochim. Acta 1999, 62, 3373.

13. Andrade, R. C. B.; Patchineelam, S. R.; Quim. Nova 2001, 23, 733.

14. Kjerfve, B.; Ribeiro, C. H. A.; Dias, G. T. M.; Filippo, A. M.; Quaresma, V. S.; Cont. Shelf Res. 1997, 17, 1609.

15. Rebello, A. L.; Haekel, W.; Moreira, I.; Santelli, R.; Schroeder, F.; Mar. Chem. 1986, 18, 215.

16. Perin, G.; Fabris, R.; Manente, S.; Wagener, A. R.; Hamacher, C.; Scotto, S.; Water Res. 1997, 12, 3017.

17. APHA; Standard methods for the examination of water and wastewater; American Public Health Association: Washington, 1995.

18. Clark, M. W.; McConchie, D. M.; Lewis, D. W.; Saenger, P.; Chem. Geol. 1998, 149, 147.

19. van den Berg, C. M. G.; Rebello, A. L.; Sci. Total Environ. 1986, 58, 37.

20. Machado, W.; Carvalho, M. F.; Santelli, R. E.; Maddock, J. E. L.; Mar. Pollut. Bull. 2004, 49, 89.

21. Silva, C. A. R.; Lacerda, L. D.; Rezende, C. E.; Biotropica 1990, 22, 339.

22. Ong Che, R. G.; Mar. Pollut. Bull. 1999, 39, 269.

23. Furukawa, K.; Wolanski, E.; Mueller, H.; Est. Coastal Shelf Sci. 1997, 44, 301.

24. Alongi, D. M.; Hydrobiologia 1994, 285, 19.

25. Borges, A. C; Sanders, C. J.; Machado, W.; Patchineelam, S. R.; Resumos do X Congresso Brasileiro de Geoquímica, Porto de Galinhas, Brasil, 2005. 